\title{
Valoración y análisis de las crisis de broncoespasmo en pacientes adultos del servicio de urgencias
}

\author{
Gianni I. Arciniega-Montiel1*, Javier Toledo-Estrada ${ }^{2}$ y Dolores G. Martínez-Marín ${ }^{3}$ \\ ${ }^{1}$ Departamento de Urgencias adultos; ${ }^{2}$ Departamento de Neumología; ${ }^{3}$ Departamento de Educación e Investigación en Salud, HGR36. Hospital \\ General de Zona 20, Instituto Mexicano del Seguro Social, Puebla, México
}

\begin{abstract}
Resumen
Introducción: El broncoespasmo es una manifestación de varias enfermedades del tracto respiratorio. La flujometría, como prueba de medición, permite valorar la funcionalidad pulmonar de forma rápida y práctica. Objetivos: Valorar y analizar las crisis agudas de broncoespasmo en pacientes adultos del servicio de urgencias del Hospital General de Zona 20, del Instituto Mexicano del Seguro Social (HGZ 20, IMSS). Material y métodos: Estudio observacional, descriptivo, transversal, prospectivo, homodémico, unicéntrico, llevado a cabo de enero a marzo 2020 en mayores de 18 años con sintomatología de broncoespasmo en el servicio de urgencias del HGZ 20, IMSS, Puebla. Se aplicó la prueba de flujometría para valoración y estadificación de la crisis de broncoespasmo. Resultados: Se incluyeron 26 pacientes, con edad promedio de 54.05, desviación estándar de \pm 18.5 años; grupo de edad más afectado, con un intervalo de 41 a 60 años. Al realizarse la medición del flujo espiratorio máximo se observó que el promedio fue de 269 l/min, con un rango de 80 a 530 l/min. El 57.6\% de los pacientes presentaron obstrucción moderada, el $26.9 \%$ leve y el $15.3 \%$ severa. La comorbilidad más asociada fue hipertensión arterial. Conclusión: Las crisis agudas de broncoespasmo pueden valorarse de forma rápida y sencilla por flujometría. Se demostró que el broncoespasmo no solo se origina por las clásicas patologías respiratorias obstructivas, sino por una extensa gama de patologías sistémicas y multifactoriales.
\end{abstract}

Palabras claves: Broncoespasmo. Flujo espiratorio máximo. Flujómetro. Flujometría. Vía aérea.

\section{Assessment and analysis of bronchospasm seizures in adult emergency department patients}

\begin{abstract}
Introduction: Bronchospasm is a manifestation of several diseases of the respiratory tract. Flowmetry as a measurement test, allows to assess lung functionality quickly and practically. Objectives: To assess and analyze acute bronchospasm crises in adult patients from the emergency department of the HGZ 20, IMSS. Material and methods: Observational, descriptive, cross-sectional, prospective, homodemic, single-center study, carried out from January to March 2020 in people over 18 years of age with symptoms of bronchospasm in the emergency service of HGZ 20, IMSS, Puebla. The flowmetry test was applied for assessment and staging of the bronchospasm crisis. Results: 26 patients were included, with a mean age of 54.05 , standard deviation of \pm 18.5 years, the most affected age group with an interval of 41 to 60 years. When the peak
\end{abstract}

\section{Correspondencia:}

*Gianni I. Arciniega-Montiel

E-mail: ali_estrella_2@msn.com
Disponible en internet: 13-09-2021

Rev Educ Investig Emer. 2021;3(3):127-132 www.medicinadeemergencias.com bajo la licencia CC BY-NC-ND (http://creativecommons.org/licenses/by-nc-nd/4.0/). 
expiratory flow was measured, it was observed that the average was $269 \mathrm{I} / \mathrm{min}$ with a range of 80 to $530 \mathrm{I} / \mathrm{min}$. $57.6 \%$ of the patients presented moderate obstruction, $26.9 \%$ mild and $15.3 \%$ severe. The most associated comorbidity was arterial hypertension. Conclusion: Acute bronchospasm attacks can be quickly and easily assessed by flowmetry. It was shown that bronchospasm is not only caused by the classic obstructive respiratory pathologies, but by a wide range of systemic and multifactorial pathologies.

Key words: Bronchospasm. Peak expiratory flow. Flow meter. Flowmetry. Airway.

\section{Introducción}

El broncoespasmo es una manifestación de varias enfermedades del tracto respiratorio como la bronquitis aguda, la agudización de enfermedad pulmonar obstructiva crónica (EPOC) y asma, neumonías, insuficiencia cardiaca y menos frecuentemente bronquiectasias infectadas e infección pleural. Se define como la contracción exagerada de la musculatura traqueobronquial como respuesta a estímulos físicos, químicos, ambientales 0 inmunitarios, generándose sibilancias, disnea, dolor y opresión torácica, tos al inhalar aire ${ }^{1}$.

La evaluación de la función pulmonar es una necesidad ineludible en las enfermedades respiratorias, principalmente en aquellas en las que la principal alteración radica en el aumento de la resistencia de la vía aérea, en el deterioro de la capacidad de difusión de los gases 0 en las alteraciones de la mecánica respiratoria.

La valoración de las crisis de broncoespasmo agudo mediante la medición del flujo espiratorio máximo (FEM), por medio del flujómetro, es una herramienta útil y accesible, además es un procedimiento fácil de utilizar para medir de manera objetiva la gravedad y el grado de obstrucción del paciente a su llegada al área de urgencias ${ }^{2}$. En comparación con la espirometría, las mediciones de flujo máximo requieren menos tiempo para su elaboración, fácil capacitación al personal y son menos costosas ${ }^{3}$.

La validación de lo que ahora se conoce como FEM de Wright comenzó a principios de la década de 1990. El FEM es el mayor flujo que se alcanza durante una maniobra de espiración forzada. Se consigue al haber expirado el $75-80 \%$ de la capacidad pulmonar total y se expresa en litros/minuto, litros/segundo o como porcentaje de su valor de referencia. Refleja el estado de las vías aéreas de gran calibre y es un índice aceptado como medida independiente de la función pulmonar'.

De acuerdo con la medición del FEM se clasifica la gravedad de la exacerbación en:

- Leve: FEM mayor del 70\% del teórico o $300 \mathrm{l} / \mathrm{min}$.

- Moderada: FEM entre el 50 y el $70 \%$ o entre 150 y $300 \mathrm{l} / \mathrm{min}$.
- Grave: FEM es inferior al 50\% o menor de $150 \mathrm{l} / \mathrm{min}^{5}$. En Chile, Orena y Valdivia encontraron que el deterioro de la función pulmonar se asocia con un FEM menor al $80 \%$. Se observó que el promedio de FEM disminuyó con la edad y se estimó una disminución promedio de $19 \mathrm{l} / \mathrm{min}$ en mujeres y $28 \mathrm{l} / \mathrm{min}$ en hombres por cada grupo de edad 6 .

Actualmente encontramos valores normales o predichos de FEM en tablas creadas en los EE.UU., que fueron validadas para la población mexicana en el Departamento de Fisiología Pulmonar del Instituto Nacional de Enfermedades Respiratorias, los cuales fueron tomados como referencias para permitir un mejor análisis y comparaciones del siguiente estudio?.

\section{Material y métodos}

Estudio observacional, descriptivo, transversal, prospectivo, homodémico, unicéntrico, efectuado en pacientes mayores de 18 años con sintomatología de broncoespasmo, a quienes se les aplicó la flujometría entre enero y marzo de 2020, sin haber recibido ningún tipo de tratamiento. La invitación e información del estudio a los participantes corrió a cargo de los médicos residentes de los servicios de urgencias, quienes recibieron previa capacitación, realizando una sola medición al ingreso del paciente al servicio de urgencias y obteniendo datos necesarios de la nota médica al ingreso. Todos los participantes aceptaron colaborar en la investigación de manera informada y voluntaria. Criterios de exclusión: pacientes con tuberculosis activa confirmada u otras enfermedades infectocontagiosas de alto riesgo, consignadas en el expediente, y pacientes con broncoespasmo de riesgo vital. Criterios de eliminación: pacientes con alta voluntaria o quienes tenían datos incompletos.

Sobre las condiciones de confidencialidad, ingreso y retiro del estudio, se utilizó un consentimiento informado y voluntario; y para la obtención de los datos demográficos y clínicos de los pacientes con broncoespasmo se aplicó un cuestionario; así mismo, se comprobó la información con la nota médica realizada a su ingreso al servicio de urgencias. 
Para la medición del FEM y valoración del grado de obstrucción se utilizó el flujómetro TruZone de tipo manual. De acuerdo con la medición del FEM clasificamos la gravedad de la exacerbación en: leve (FEM mayor del $70 \%$ del teórico o $300 \mathrm{l} / \mathrm{min}$ ), moderada (FEM entre el 50 y el $70 \%$ o entre 150 y $300 \mathrm{l} / \mathrm{min}$ ) y grave (FEM inferior al $50 \%$ o menor de $150 \mathrm{l} / \mathrm{min})^{5}$.

\section{Análisis estadístico}

Para el estudio estadístico se utilizó el programa SPSS v 25 para Mac. El análisis descriptivo consistió en frecuencias y porcentajes para variables nominales $u$ ordinales. Para las variables cuantitativas se utilizo la media, mínima y máxima, y desviación estándar.

\section{Consideraciones éticas}

El estudio fue sometido y aprobado por el comité local de investigación y ética en salud, con número de registro R-2019-2106-042, de riesgo mínimo, apegado en todo momento al Reglamento de la Ley General de Salud en Materia de Investigación en Salud y a la declaración de Helsinki modificada por la 64. ${ }^{\text {a }}$ Asamblea General de Fortaleza, Brasil, en octubre del 2013, y a las normas éticas internacionales y a las normas institucionales relacionadas con la investigación científica.

\section{Resultados}

Se estudiaron 26 pacientes que presentaron crisis de broncoespasmo, en los cuales no hubo exclusión, 14 hombres y 12 mujeres con rango de edad de 20 a 84 años, edad promedio de 54.05 , con una desviación estándar de \pm 18.5 años. Se identificó que 10 pacientes (38.4\%) estaban en normopeso, 8 (30.7\%) en sobrepeso, $5(19.2 \%)$ en obesidad grado I y $3(11.5 \%)$ en obesidad grado II.

Las comorbilidades mas frecuentes fueron: un $30.7 \%$ con hipertensión arterial sistémica, un 30.7\% con insuficiencia cardiaca, un $11.5 \%$ con asma y enfermedad pulmonar intersticial difusa, un $7.6 \%$ con cáncer de próstata, enfermedad renal crónica y enfermedad pulmonar obstructiva crónica, y un $3.8 \%$ con cáncer testicular y síndrome de apnea obstructiva del sueño.

De los síntomas asociados que presentaron los pacientes con crisis de broncoespasmo agudo, se encontró que el $100 \%$ de los pacientes presentaron
Tabla 1. Grado de obstrucción que presentaron los pacientes del Servicio de Urgencias del Hospital General de Zona N. ${ }^{\circ} 20$, de acuerdo con el flujo espiratorio máximo medido por flujometría $(n=26)$

\begin{tabular}{|l|c|c|}
\hline Grado de obstrucción & Frecuencia & Porcentajes \\
\hline Leve & 7 & $26.9 \%$ \\
\hline Moderado & 15 & $57.6 \%$ \\
\hline Severo & 4 & $15.3 \%$ \\
\hline
\end{tabular}

Tabla 2. Valores de flujo espiratorio máximo (FEM) que presentaron los pacientes con crisis de broncoespasmo agudo medido por flujometría $(n=26)$

\begin{tabular}{|c|c|c|}
\hline FEM por rango & Número de pacientes & $\%$ \\
\hline 501 a $600 \mathrm{l} / \mathrm{min}$ & 1 & $3.8 \%$ \\
\hline 401 a $500 \mathrm{l} / \mathrm{min}$ & 3 & $11.5 \%$ \\
\hline 301 a $400 \mathrm{l} / \mathrm{min}$ & 3 & $11.5 \%$ \\
\hline 201 a $300 \mathrm{l} / \mathrm{min}$ & 14 & $53.8 \%$ \\
\hline 101 a $200 \mathrm{l} / \mathrm{min}$ & 4 & $15.3 \%$ \\
\hline 50 a $100 \mathrm{l} / \mathrm{min}$ & 1 & $3.8 \%$ \\
\hline
\end{tabular}

sibilancias, el $80.7 \%$ disnea, el $57.6 \%$ tiraje intercostal, el $46.1 \%$ dolor torácico, el $38.4 \%$ disociación toracoabdominal, el $30.7 \%$ tos y el $23.07 \%$ expectoración. El $57.6 \%$ presentaron obstrucción moderada, el $26.9 \%$ leve y el $15.3 \%$ severa (Tabla 1).

En el análisis del FEM que presentaron los pacientes con crisis de broncoespasmo agudo medido por flujometría, se encontró: 1 paciente en el rango de 501 a $600 \mathrm{l} / \mathrm{min}, 3$ pacientes en el rango de 401 a $500 \mathrm{l} / \mathrm{min}$, 3 en el rango 301 a $400 \mathrm{l} / \mathrm{min}, 14$ en el rango de 201 a $300 \mathrm{l} / \mathrm{min}, 4$ en 101 a $200 \mathrm{l} / \mathrm{min}$ y 1 en el rango de 50 a $100 \mathrm{l} / \mathrm{min}$ (Tabla 2).

Los diagnósticos de egreso finales de los pacientes con crisis de broncoespasmo agudo fueron en el $19.2 \%$ de los casos insuficiencia cardiaca, el 19.2\% de tromboembolia pulmonar, el $11.5 \%$ de metástasis a pulmón, asma controlada y enfermedad pulmonar intersticial difusa, el $7.6 \%$ de infección de vías respiratorias, enfermedad pulmonar obstructiva crónica y edema agudo pulmonar y el 3.8\% de enfermedad por reflujo gastroesofágico (Tabla 3).

Los pacientes con crisis de broncoespasmo agudo tuvieron como destino en el $61.5 \%$ de los casos ingreso al piso de medicina interna y el $38.4 \%$ fueron egresados 
Tabla 3. Diagnósticos de egreso finales que presentaron los pacientes con crisis de broncoespasmo $(n=26)$

\begin{tabular}{|l|c|c|}
\hline Diagnostico final & $\begin{array}{c}\text { Número de } \\
\text { pacientes }\end{array}$ & Porcentajes \\
\hline Insuficiencia cardiaca & 5 & $19.2 \%$ \\
\hline Tromboembolia pulmonar & 5 & $19.2 \%$ \\
\hline Metástasis a pulmón & 3 & $11.5 \%$ \\
\hline Asma controlada & 3 & $11.5 \%$ \\
\hline $\begin{array}{l}\text { Enfermedad pulmonar } \\
\text { intersticial difusa }\end{array}$ & 3 & $11.5 \%$ \\
\hline $\begin{array}{l}\text { Infección de vías } \\
\text { respiratorias }\end{array}$ & 2 & $7.6 \%$ \\
\hline EPOC & 2 & $7.6 \%$ \\
\hline Edema agudo de pulmón & 2 & $7.6 \%$ \\
\hline Enfermedad por reflujo & 1 & $3.8 \%$ \\
\hline gastroesofágico & & \\
\hline EPOC: enfermedad pulmonar obstructiva crónica. & \\
\hline
\end{tabular}

sin realización de flujometría a sus domicilios con mejoría clínica y tratamiento especializado.

\section{Discusión}

Las principales causas mundiales de crisis de broncoespasmo agudo son asma y EPOC. Estudios previos realizados en Guangdong, China, evaluaron si un medidor de flujo máximo manual era efectivo como herramienta para la detección de obstrucción del flujo de aire; en ese estudio la mayoría de las crisis se debió a la EPOC ${ }^{8}$. En nuestro estudio encontramos como causas más frecuentes de broncoespasmo a la insuficiencia cardiaca y la tromboembolia pulmonar, en el $19.2 \%$ de los casos.

Se encontró que la población con crisis de broncoespasmo agudo que acuden al servicio de urgencias suele estar más presente en hombres (en un 53.8\%) con respecto a las mujeres (en un $45.1 \%$ ). El grupo de edad más afectado se encuentra en un intervalo de 41 a 60 años.

En un estudio realizado en Chile por Orena-Valdivia en 2018 se obtuvieron como valores promedios de FEM $330 \mathrm{l} / \mathrm{min}$ en mujeres y $460 \mathrm{l} / \mathrm{min}$ en hombres; con diferencias según edad, educación, índice de masa corporal y actividad física ${ }^{9}$. En nuestro estudio se observaron valores promedio de FEM comparativamente bajos, de $273 \mathrm{l} / \mathrm{min}$ en mujeres y $265 \mathrm{l} / \mathrm{min}$ en hombres.
Tabla 4. Grado de obstrucción que presentaron los pacientes con crisis de broncoespasmo, según sexo $(\mathrm{n}=26)$

\begin{tabular}{|l|l|c|c|}
\hline Sexo & $\begin{array}{l}\text { Grado de } \\
\text { obstrucción }\end{array}$ & $\begin{array}{c}\text { Número de } \\
\text { pacientes }\end{array}$ & $\%$ \\
\hline \multirow{3}{*}{ Femenino } & Leve & 4 & $15.3 \%$ \\
& Moderado & 6 & $23 \%$ \\
& Severo & 2 & $7.6 \%$ \\
\hline \multirow{3}{*}{ Masculino } & Leve & 3 & $11.5 \%$ \\
& Moderado & 9 & $34.6 \%$ \\
& Severo & 2 & $7.6 \%$ \\
\hline
\end{tabular}

En la investigación realizada en Lima, Perú, por Montenegro-Banalcazar en 2017, en población de 20 a 60 años, el FEM promedio fue de $112 \mathrm{l} /$ min en mujeres y 517 I/min en varones, el FEM disminuyó significativamente conforme aumentó la edad en ambos sexos, el peso no determinó diferencia en el FEM ${ }^{10}$.

Por su parte, el promedio de FEM observado tanto en hombres como en mujeres fue inferior al valor predicho creado en los EE.UU., validado para la población mexicana en el Departamento de Fisiología Pulmonar del Instituto Nacional de Enfermedades Respiratorias. Una posible explicación a este resultado podría ser la existencia de diversos factores que pueden afectar el FEM en las poblaciones: sexo, edad, talla y técnica de la maniobra son los más reconocidos ${ }^{10}$.

Las comorbilidades más frecuentes asociadas a la crisis de broncoespasmo en nuestra población fueron la hipertensión arterial, seguida de la insuficiencia cardiaca, asma y enfermedad pulmonar intersticial difusa.

Otra diferencia significativa hallada en nuestra población fue lo observado respecto al grado de obstrucción, las crisis moderadas fueron las que predominaron, presentándose en un $57.6 \%$, siendo los hombres quienes se encontraron en este rango en un $34.6 \%$. En el grado de obstrucción severa no hubo diferencia de acuerdo con el sexo (Tabla 4). En comparación con la población estudiada en el Hospital León Becerra, Guayaquil, Ecuador, donde el $72 \%$ de las crisis fueron en pacientes masculinos y el $28 \%$ femeninos. La gravedad de la crisis fue: leve $76 \%$, moderada $20 \%$ y severa $4 \%{ }^{11}$.

Es importante mencionar que los estudios realizados sobre el uso y valoración de la flujometría están enfocados solamente a patologías respiratorias obstructivas como asma y EPOC y no se encuentran 
estudios en la literatura que comparen los hallazgos de flujometría en patologías diferentes a las obstructivas. En la actualidad, la flujometría no se toma en cuenta para la valoración, tratamiento y seguimiento de los pacientes con crisis de broncoespasmo agudo en los servicios de urgencias del país e incluso a nivel mundial, a pesar de su utilidad y sencillez demostrada para la medición objetiva de la función pulmonar y en particular para el monitoreo y seguimiento del broncoespasmo.

Por hallazgos de este estudio se recomienda utilizar la flujometría para la valoración de la mayoría de las crisis de broncoespasmo, excepto las que comprometen la vida; se requiere una breve capacitación, conocimiento, práctica con el equipo y la técnica adecuada, así como una valoración oportuna del FEM al ingreso a la sala de urgencias.

Se debe recomendar ampliamente el apego a las guías y avances médicos internacionales en todos los servicios de urgencias, teniendo en cuenta los recursos y limitaciones de cada unidad hospitalaria. En lo que respecta a la valoración y atención de los pacientes con sintomatología respiratoria, en particular el broncoespasmo agudo, urge establecer a la flujometría como recurso y herramienta fundamental para el diagnóstico inicial básico, siendo un instrumento de medición sencillo, económico, certero e inocuo, es indispensable que todo médico de urgencias conozca su utilidad. Los departamentos de emergencias deben tomar medidas para fomentar el acceso a los medidores de flujo máximo para que los clínicos y el personal de enfermería puedan evaluar con mayor precisión a los pacientes y monitorear la respuesta al tratamiento.

\section{Fortalezas y limitaciones}

Es importante mencionar que debido a la pandemia por enfermedad por coronavirus 2019 (COVID-19), se tuvo que detener el estudio a partir del ingreso de los primeros casos sospechosos en el HGZ 20, esto derivado del alto riesgo de trasmisión por la liberación de aerosoles al momento de aplicar la flujometría; no obstante que la COVID-19 presenta importantes manifestaciones respiratorias, incluyendo el broncoespasmo. Por lo que el tiempo durante el cual se reclutaron pacientes representa un factor limitante de nuestro estudio. Futuras investigaciones deberán incluir un tamaño de muestra más amplio y representativo.

\section{Conclusión}

Las crisis agudas de broncoespasmo pueden ser valoradas de forma rápida y sencilla por flujometría. Se demostró que el broncoespasmo no solo se origina por las clásicas patologías respiratorias obstructivas, sino por una extensa gama de patologías sistémicas y multifactoriales. La medición y valoración de la flujometría en urgencias debe considerarse un estudio de extensión de gran apoyo para el médico de urgencias en la toma de decisiones para evitar complicaciones irreversibles.

\section{Financiamiento}

La presente investigación no ha recibido ninguna beca específica de agencias de los sectores públicos, comercial o sin ánimo de lucro.

\section{Conflicto de intereses}

Los autores declaran no tener conflicto de intereses.

\section{Responsabilidades éticas}

Protección de personas y animales. Los autores declaran que los procedimientos seguidos se conformaron a las normas éticas del comité de experimentación humana responsable y de acuerdo con la Asociación Médica Mundial y la Declaración de Helsinki.

Confidencialidad de los datos. Los autores declaran que han seguido los protocolos de su centro de trabajo sobre la publicación de datos de pacientes.

Derecho a la privacidad y consentimiento informado. Los autores han obtenido el consentimiento informado de los pacientes y/o sujetos referidos en el artículo. Este documento obra en poder del autor de correspondencia.

\section{Bibliografía}

1. Becerra J, Fernández J, Rueda C. Broncoespasmo. Málaga, España: 2000. Disponible en: http://www.medynet.com/usuarios/jraguilarManual\%20de\%20urgencias\%20y\%20Emergencias/broncoes.pdf

2. Hidalgo $P$, Puga M, Vargas J. Uso del flujo espiratorio máximo (FEM) para clasificar los niveles de gravedad de los pacientes con crisis asmática que acuden al servicio de urgencias [tesis doctoral en internet]. [Santiago de Guayaquil (EC)]: Universidad Católica de Santiago de Guayaquil; 2010. Disponible en: http://repositorio.ucsg.edu.ec/handle/3317/608

3. Veloz-Montenegro M, Benalcázar-Game J, Domínguez-Bernita E. Algunas consideraciones sobre el examen de pico flujo y su medición. Rev Cient Dominio Cienc. 2017;3:177-87.

4. Comité Ejecutivo de la GEMA. GEMA 4.3 Guía Española para el Manejo del Asma [Internet]. Madrid, España: Luzán 5; 2017. Disponible en: https://www.semg.es/images/documentos/docs_varios/GEMA_43.pdf 
Rev Educ Investig Emer. 2021;3(3)

5. Rodrigo GJ, Plaza Moral V, Bardagí Forns S, Castro-Rodríguez JA, De Diego Damiá A, Liñán Cortés $S$, et al. Guía ALERTA 2. América Latina y España: Recomendaciones para la prevención y el tratamiento de la exacerbación asmática. Arch Bronconeumol. 2010;46:2-20.

6. Orena V, Valdivia G, Ferreccio C. Flujo espiratorio máximo: caracterización en un estudio en población adulta chilena; resultados basales de la cohorte del Maule (MAUCo). Rev Chil Enferm Respir. 2018;34:212-22.

7. Sociedad Mexicana de Neumología y Cirugía de Tórax. Consenso Mexicano del asma. Neumol Cir Torax [Internet]. 2005;64(Supl 1):S7-S44 Disponible en: https://www.medigraphic.com/pdfs/neumo/nt-2005/ nts051c.pdf
8. Tian J, Zhou Y, Cui J, Wang D, Wang X, Hu G, et al. Peak expiratory flow as a screening tool to detect airflow obstruction in a primary health care setting. Int J Tuberc Lung Dis. 2012;16:674-80.

9. Thorat $Y$, Salvi $S$, Kodgule $R$. Peak flow meter with a questionnaire and mini-spirometer to help detect asthma and COPD in real-life clinical practice: across-sectional study. NPJ Prim Care Respir Med. 2017;27:s32.

10. Montenegro M, Benalcázar J, Domínguez E. Algunas consideraciones sobre e examen de pico flujo y su medición. Rev Cient Dominio Cienc. 2017:3:177-87.

11. So J, Lastra A, Zhao H, Marchetti N, Criner GJ. Daily peak expiratory flow rate and disease instability in chronic obstructive pulmonary disease. Chronic Obstr Pulm Dis. 2016;3:398-405. 